\title{
A thermal insulation method for a piezoelectric transducer
}

\author{
WU Song, CHEN Hong, GU JiaHua \& YU HongRu ${ }^{\dagger}$ \\ Key Laboratory of High Temperature Gas Dynamics, Institute of Mechanics, Chinese Academy of Sciences, Beijing 100080, China
}

This study deals with the sources of signal distortion of a piezoelectric transducer heated by measured gas flow. These signal distortions originate from both unloading of preload on a piezocrystal because of expansion of a diaphragm in the test apparatus and the pyroelectric effect of a heated piezoelectric crystal. A plastic film on the diaphragm of the transducer can effectively insulate the diaphragm and the piezocrystal within transducer from heating by gas flow, eliminating the sources of distortion. A method for evaluating the thickness of the film is proposed.

instantaneous pressure measurement, piezoelectric transducer, thermal insulation

Piezoelectric transducers have been utilized extensively for measuring dynamic pressure because of their high-frequency responses and wide measuring ranges. During measurements in hypervelocity flow or in a shock wave, blast wave, or detonation wave, a piezoelectric transducer is heated simultaneously by gas flow and is affected by pressure. The output signal is thus generated by both piezoelectric and pyroelectric effects of the piezoelectric crystal. The mixed signal is difficult to deconvolute, which leads to significant distortion of the results. In order to surmount this difficult problem, the sources of thermal interaction with a piezoelectric transducer are first analyzed. Based on good grasp of thermal interactions with the transducer, a convenient and effective thermal insulation scheme and criteria for insulation selection are proposed.

\section{Sources of thermal interaction for a piezoelectric transducer}

The measuring sensor of a piezoelectric transducer is a piezoelectric crystal. While load acts on the crystal, the induced charge increases as the pressure is increased, owing to the piezoelectric effect of the crystal. In addition, the crystal possesses a pyroelectric effect, i.e., when the crystal is heated, induced charge increases with increasing temperature of the crystal. An immediate way to eliminate the thermal interaction with a piezoelectric transducer is to insulate the crystal from the source of heat. We first tried to insert a piece of ceramic between the diaphragm and the crystal as an insulator (Figure 1), but measured data indicated that the distortion signal was not eliminated. Analysis of the experimental results indicated that the thermal interaction must have yet another source.

The piezoelectric transducer usually has upon it an exerted preload, i.e., the crystal undergoes a certain deformation even if no load is applied to the transducer. When the diaphragm of the transducer is heated, the preload on the crystal drops because of expansion of the diaphragm, which leads to a decreased induced charge. It is evident that the induced charges caused by heating a transducer may be classified as two kinds. One is produced by the pyroelectric effect of heated crystal and another is produced by unloading of preload on crystal. These two kinds of induced charge have opposite polarity. The expansion of diaphragm leads to decreasing of

Received February 12, 2007; accepted March 10, 2007

doi: 10.1007/s11434-007-0366-y

'Corresponding author (email: yuhr@imech.ac.cn)

Supported by the National Natural Science Foundation of China (Grant No. 90605006) 
the charge, but the temperature increase of the crystal leads to increasing of the charge. Furthermore, heat is transferred into the diaphragm first; it then conducts to the crystal through the diaphragm. The sequence of temperature rise of both the diaphragm and the crystal is different. The unloading signal due to diaphragm expansion appears first, and afterwards a similar loading signal caused by increasing temperature of piezoelectric crystal accumulates.

To verify whether the above speculation is right or wrong, an experiment was designed and conducted. Both a piezoelectric transducer and a platinum-film resistance thermometer were immersed simultaneously into hot water $\left(60^{\circ} \mathrm{C}\right)$. Voltage $(V)$ vs. time $(t)$ data is shown in Figure 2. The upper curve $(T)$ is the output of the resistance thermometer, which approaches a parabola. According to the principle of heat conduction ${ }^{[1]}$, the heat transfer rate is nearly constant. The lower one $(P)$ is the output of the piezoelectric transducer. As shown, a negative jump in the signal appears immediately after im-

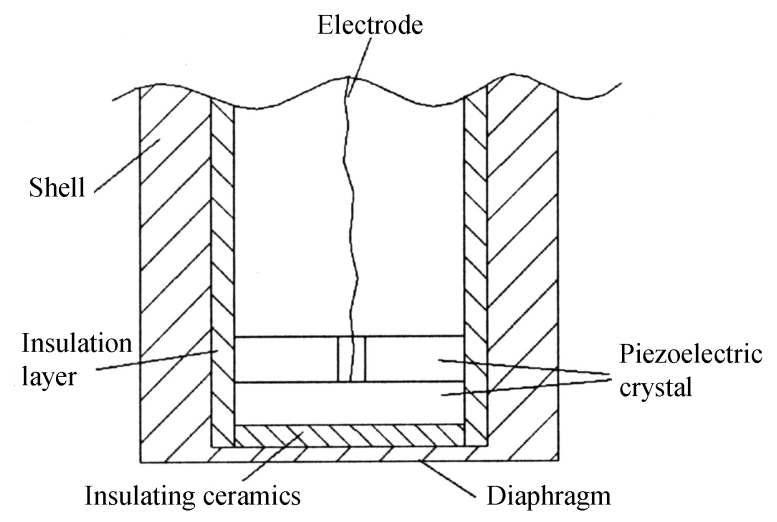

Figure 1 Schematic diagram of piezoelectric transducer with a transducer ceramic insulator.

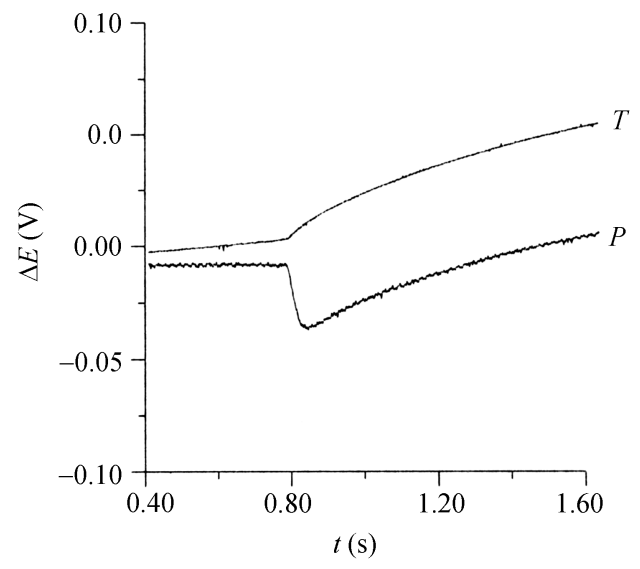

Figure 2 Output curves of thermometer structure and piezoelectric transducer. mersion, because the diaphragm is heated first. Because a positive signal produced by continuous increasing temperature of the piezoelectric crystal is accumulated on the output signal, the drop is followed by a climbing section.

The experimental results provide verification of the above-mentioned tentative idea.

\section{Insulation method}

As discussed, the distortion signals of a piezoelectric transducer due to heating by gas flow have origins both in diaphragm expansion and in increasing temperature of the piezoelectric crystal. Furthermore, the heat is transferred first to the diaphragm, and by way of the diaphragm the heat is conducted into the piezoelectric crystal. Therefore, the unloading signal as a result of diaphragm expansion appears first, and a similar loading signal which results from the increasing temperature of crystal accumulates afterwards. Although heating of the diaphragm and the crystal of a piezoelectric transducer each produce distortion signals, only the diaphragm is in need of thermal insulation from the gas flow in an instantaneous measurement.

Any insulation scheme that is imposed requires not only effective shielding from the heat imparted by the gas flow, but also as small as possible variation in the transducer performance (such as sensitivity, response frequency, etc.). Attempts to insulate the transducer from heating include coating with grease on the diaphragm of the transducer, filling oil into the measuring hole or insufflating gas, and positioning a piece of plastic film over the surface of the diaphragm. For avoiding pollution or other disturbance of the gas flow and for easy control, to place a plastic film on the diaphragm has proved to be the simplest and most effective insulation method.

\section{Material selection and thickness evaluation of insulating film}

The plastic film is a flat sheet of uniform thickness. There are thus only two parameters to be decided: material type and film thickness.

The extent of the signal distortion depends mainly on the magnitude of unloading of the preload on the crystal. This magnitude is related to the heat input into the diaphragm, and the structure, material, and assembling 
process of the transducer. It is more than extremely complicated to evaluate the unloading magnitude from the heat transferred into the diaphragm; it is impossible because the required data cannot be obtained. In order to surmount this difficulty, another approach was proposed: the temperature rise of the diaphragm would be limited within an allowable range within the duration of the measurement. Under this condition, it may be regarded that the distortion may be ignored. As will be shown later, the temperature rise of diaphragm is easy to limit to an extremely low level during the instantaneous measurements.

\subsection{Surface temperature rise of the diaphragm}

In the case of the surface of a flat plate for which heat is transferred by steady heat flux $q_{0}$ and its back is insulated, the temperature rise in flat plate can be represented as follows:

$$
\begin{aligned}
\Delta T(y)= & \frac{2 q_{0} \sqrt{\alpha t}}{K} \sum_{n=0}^{\infty}\left\{\operatorname{ierfc} \frac{(2 n+1) \ell-y}{2 \sqrt{\alpha t}}\right. \\
& \left.+\operatorname{ierfc} \frac{(2 n+1) \ell+y}{2 \sqrt{\alpha t}}\right\}
\end{aligned}
$$

temperature rise of the surface is

$$
\begin{aligned}
\Delta T_{f}(y=\ell)= & \frac{2 q_{0} \sqrt{\alpha t}}{K} \sum_{n=0}^{\infty}\left\{\operatorname{ierfc} 0+2 \operatorname{ierfc} \frac{\ell}{\sqrt{\alpha t}}\right. \\
& \left.+2 \operatorname{ierfc} \frac{2 \ell}{\sqrt{\alpha t}}+2 \operatorname{ierfc} \frac{3 \ell}{\sqrt{\alpha t}}+\cdots\right\} ;
\end{aligned}
$$

and the temperature rise of the back is

$$
\begin{aligned}
\Delta T_{b}(y=0)= & \frac{2 q_{0} \sqrt{\alpha t}}{K} \sum_{n=0}^{\infty}\left\{2 i e r f c \frac{\ell}{2 \sqrt{\alpha t}}\right. \\
& \left.+2 \operatorname{ierfc} \frac{3 \ell}{2 \sqrt{\alpha t}}+2 i e r f c \frac{5 \ell}{2 \sqrt{\alpha t}}+\cdots\right\} ;
\end{aligned}
$$

where $\alpha=\frac{K}{\rho C}, K, t$, and $\ell$ are thermal diffusivity, thermal conductivity, heating time, and thickness, respectively.

The temperature increases on the front and rear surfaces of a flat plate are shown in Figure 3. The solid curves in this figure are calculated according to formulae (2) and (3). The dashed ones are the surface temperature increases of the plate with a substrate ${ }^{[2]}$. It can be seen that the front surface temperature rises of the plate, both with and without substrate, are nearly the same, to long times after being heated. But the rear surface temperature rise of the plate with substrate is lower than that of the plate without substrate. The front surface temperature of flat plate increases quickly at the early stage. In contrast with front surface, the rear surface temperature increases more slowly.

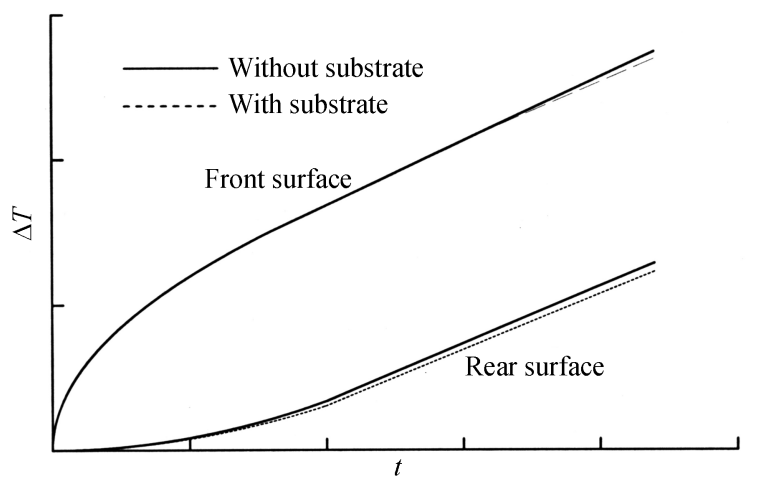

Figure 3 Variations of front and rear surface temperature rise of flat plate with time.

The structure of diaphragm covered by an insulating film is sketched in Figure 4 (assuming the insulating film is contacted completely with diaphragm of transducer). So the surface temperature of diaphragm is equal to the rear surface temperature of the insulating film. Further, assuming the temperature increase of the film without substrate is adopted, the two suppositions are stricter than the practice.

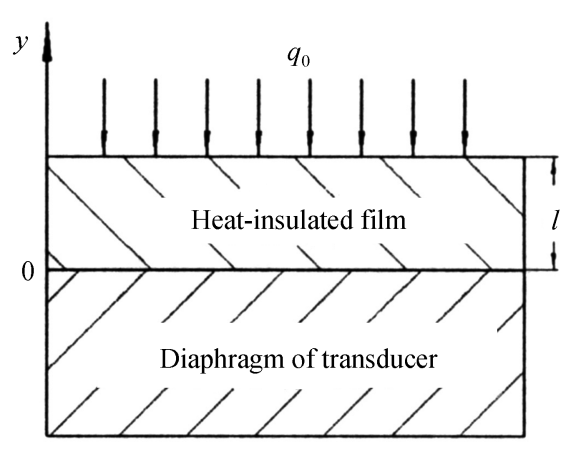

Figure 4 Model for heat transfer in diaphragm covered by a film.

While the diaphragm of a transducer is exposed directly to gas flow, its surface temperature increases rapidly. If the diaphragm is covered by a piece of thermal insulation, the rate of temperature rise of diaphragm surface should become very slow.

The level of signal distortion can be expressed by the surface temperature rise of the diaphragm. The higher the temperature rise, the more distortion results we will get. According to formula (3), $\Delta T_{b}$ is in proportion to $q_{0}$ and rapidly decreases with increasing $\ell / \sqrt{\alpha t}$. 


\subsection{Material selection of the insulating film}

Thermal diffusivity is an intrinsic parameter of the film material. The surface temperature rise of diaphragm decreases with decreasing thermal diffusivity. Thus, the thermal diffusivity of film material is chosen to be as small as possible. Teflon has thermal diffusivity of $1.14 \times$ $10^{-7} \mathrm{~m} / \mathrm{s}$, which makes it a suitable choice.

\subsection{Thickness evaluation of the insulating film}

On conditions that Teflon $\left(\alpha=1.14 \times 10^{-7} \mathrm{~m}^{2} / \mathrm{s}\right.$, $\left.K=0.24 \mathrm{~W} \cdot \mathrm{K}^{[3]}\right)$ is selected as the insulating film and constant heat flux $q_{0}=1 \mathrm{MW} / \mathrm{m}^{2}$ into the front surface of the film, the temperature rise of the rear surface of film (i.e., temperature rise of transducer diaphragm) can be calculated according to formula (3). Calculated results are shown in Table 1.

Table 1

\begin{tabular}{lcccc}
\hline$\ell(\mathrm{mm})$ & 0.05 & 0.1 & 0.2 & 0.2 \\
\hline$t(\mathrm{~ms})$ & 10 & 10 & 10 & 20 \\
$\ell / \sqrt{\alpha t}$ & 1.48 & 2.96 & 5.92 & 4.18 \\
$\Delta T_{\mathrm{b} \max }(\mathrm{K})$ & 60 & 5 & 0.002 & 0.4 \\
\hline
\end{tabular}

It can be seen from the table that the temperature increases of the rear surface are sensitive to the film thickness and the measurement duration. For measurement duration of $10 \mathrm{~ms}$, when the film thickness increases from 0.1 to $0.2 \mathrm{~mm}$, the temperature increase drops by $1 / 2500$. If the film thickness is fixed at $0.2 \mathrm{~mm}$ and the measurement duration increases from 10 to 20 $\mathrm{ms}$, the temperature increase rises by 200 times.

Provided the plastic is thin and compliant, an insulating film placed over a diaphragm should have no influence on the properties of the transducer.

\section{Experimental verification}

A detonation can generate high temperatures, high pressures, and high-speed gas flow, and the pressure field behind a detonation wave can be predicted. A detonation tube is therefore adapted as the facility for experimental verification. The structure and wave diagram of the detonation tube are illustrated in Figure $5^{[4]}$. The igniter is placed at the left end of the tube. Both the piezoelectric transducers with and without a thermally insulating film are installed at the same section, which is $1.55 \mathrm{~m}$ from the left end. A thin diaphragm is used to separate the detonation section and dumping section. The pres- sure of the detonatable mixture is $0.2 \mathrm{MPa}$ and the mixture ratio of hydrogen to oxygen to nitrogen is $2: 1: 1$.

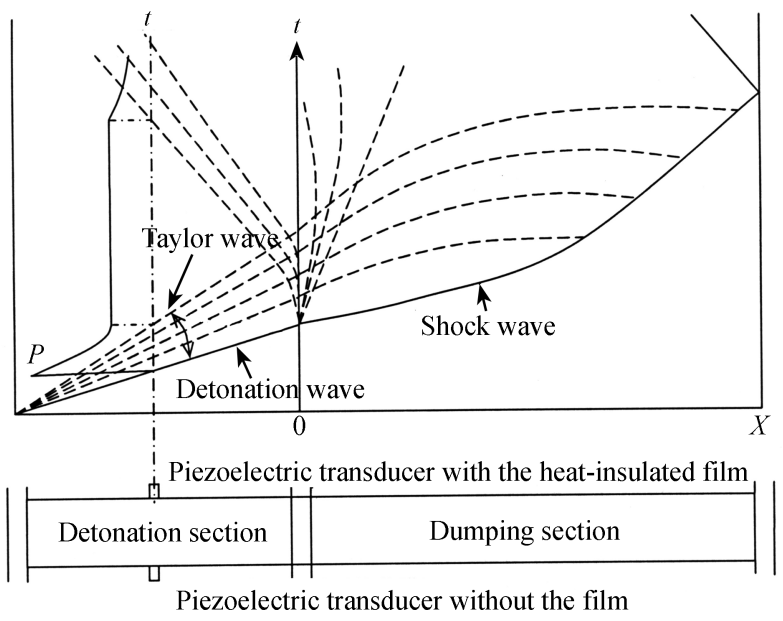

Figure 5 Structure and wave diagram of detonation tube.

The arrival of detonation front makes the pressure jump steeply. The pressure then gradually drops because the Taylor wave behind detonation wave sweeps through. After that, the pressure remains constant for 5-6 ms. The expansion wave produced by the diaphragm rupture then arrives and the pressure again drops.

Figure 6 shows the measured results of the piezoelectric transducers with and without an insulating film, along with the theoretical prediction. The noisy curves are the measured data and the smooth curve is the theoretical prediction. It is evident that there is significant difference between the prediction and the data measured by the transducer without the insulating Teflon film. In contrast, the measured results of the transducer covered by a Teflon film of $0.13 \mathrm{~mm}$ thickness are in excellent agreement with the prediction. Not only the pressure peak but also the duration of platform is consistent.

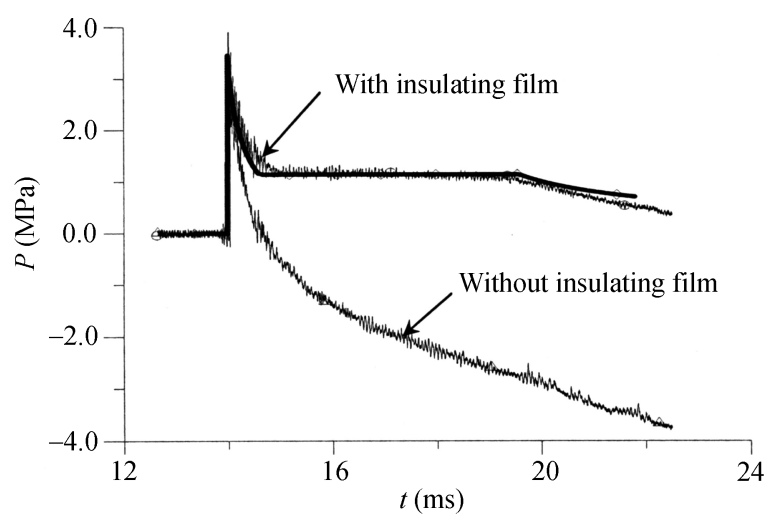

Figure 6 Pressure variation of gas flow generated by detonation. 
These results illustrate that the thermal interaction of the transducer can be significant and that a $0.13 \mathrm{~mm}$ thick Teflon film is an outstanding insulator under these conditions.

\section{Conclusions}

Placing a thin piece of Teflon on the front diaphragm of a piezoelectric transducer proved to be a most convenient and efficient way to insulate the transducer from heating by a measured gas flow.

1 Carslaw H S, Jaeger J C. Conduction of Heat in Solids. Oxford: Claredon Press, 1986

2 Schultz D L, Jones T V. Heat-transfer measurements in short-duration hypersonic facilities. AGARDograph Monograph No. 165, 1973
Given the allowable maximum temperature rise of diaphragm, heat flux transferred to diaphragm, and measurement duration, the required thickness of the insulating film can be calculated. The effectiveness of the insulating film of a given thickness was verified experimentally. This thickness of film can be applied in practice, and can be certified to meet the requirements of heat isolation. When the measurement conditions are different from those in the verification experiment, the thickness of the insulating film can be adjusted according to formula (3).

3 Shen X H. The Manual of Seal Material (in Chinese). Beijing: Chinese Petrochemical Press, 1991. 170-178

4 Chen H, Feng H, Yu H R. Double detonation drivers for a shock tube/tunnel. Sci China Ser. G-Phys, Mech, Astron, 2004, 47(4): 502—512 